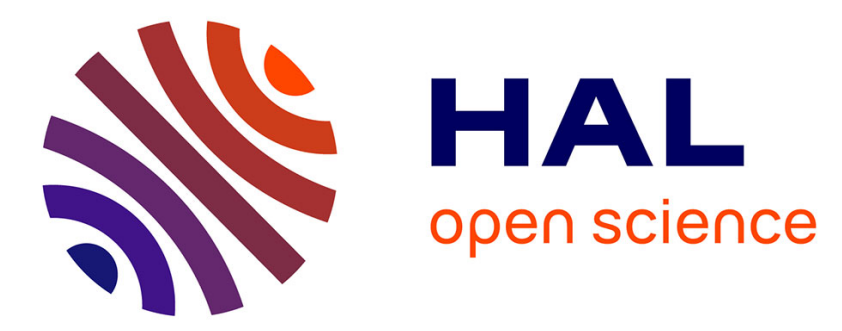

\title{
Prise en charge des anomalies intracrâniennes de découverte fortuite
}

D. Leys, N. Hadhoum, J.-P. Lejeune, J.-P. Pruvo

\section{To cite this version:}

D. Leys, N. Hadhoum, J.-P. Lejeune, J.-P. Pruvo. Prise en charge des anomalies intracrâniennes de découverte fortuite. Pratique Neurologique - FMC, 2019, 10, pp.71 - 76. 10.1016/j.praneu.2019.02.004 . hal-03486517

\section{HAL Id: hal-03486517 https://hal.science/hal-03486517}

Submitted on 20 Dec 2021

HAL is a multi-disciplinary open access archive for the deposit and dissemination of scientific research documents, whether they are published or not. The documents may come from teaching and research institutions in France or abroad, or from public or private research centers.
L'archive ouverte pluridisciplinaire HAL, est destinée au dépôt et à la diffusion de documents scientifiques de niveau recherche, publiés ou non, émanant des établissements d'enseignement et de recherche français ou étrangers, des laboratoires publics ou privés.

\section{다)(1) $(5$}

Distributed under a Creative Commons Attribution - NonCommercial| 4.0 International 


\section{Prise en charge des anomalies intracrâniennes de découverte fortuite.}

\section{Management of coincidental intracranial abnormalities.}

Didier LEYS ${ }^{1}$ *, Nawal HADHOUM ${ }^{2}$, Jean-Paul LEJEUNE ${ }^{3}$, Jean-Pierre PRUVO ${ }^{4}$.

${ }^{1}$ Univ-Lille, INSERM U 1171, CHU Lille. Service de neurologie vasculaire, 59037 Lille

${ }^{2}$ Univ-Lille, INSERM U 995. CHU Lille. Service de neurologie inflammatoire, 59037 Lille

${ }^{3}$ Univ-Lille, Inserm U 1189. CHU Lille. Service de neurochirurgie, 59037 Lille

${ }^{4}$ Univ-Lille, INSERM U 1171. CHU Lille. Service de neuroradiologie, 59037 Lille

*Correspondance : Professeur Didier LEYS. Service de Neurologie et Pathologie

Neurovasculaire. CHU de Lille. Hôpital Roger Salengro. Rue Emile Laine. 59037 Lille.

Tel 03204468 13. Fax : 0310446028.

E-mail : didier.leys@univ-lille.fr 


\title{
Résumé
}

La découverte fortuite d'anomalies lors d'une exploration en imagerie par résonance magnétique (IRM) est observée 1 fois toutes les 37 IRM si l'on exclue les anomalies de la substance blanche, les infarctus silencieux et les micro-saignements. Cette proportion augmente avec l'injection de gadolinium, des séquences angiographiques, et l'âge des personnes.

Les conséquences sont parfois bénéfiques pour le patient lorsqu'elles permettent un traitement (par exemple d'une tumeur), ou la surveillance d'une lésion à risque évolutif (syndrome radiologique isolé susceptible de représenter un stade précoce de sclérose en plaques). Elles sont plus souvent délétères par les conséquences psychologiques et sociales qu'elles induisent, et les stratégies thérapeutiques auxquelles elles exposent, soient en traitant des anomalies qui ne devraient pas l'être (par exemple certaines malformations artério-veineuses), ou en privant par excès de prudence le patient d'un traitement (thrombolyse intraveineuse en présence d'un anévrisme silencieux). Dans la plupart des cas l'incertitude prévaut et la décision nécessite, en l'absence d'essais randomisés, une analyse multidisciplinaire du risque spontané et du traitement, et la préférence du patient.

Un médecin qui demande la réalisation d'une IRM doit avoir à l'esprit le risque de découverte d'une anomalie fortuite qui ne rendra qu'exceptionnellement service au patient. Cette découverte peut générer de l'anxiété chez les patients et leurs proches, et les exposer à des refus d'assurances, et une consommation excessive et souvent injustifiée de ressources médicales. Une telle éventualité doit être anticipée, ce qui serait plus aisé si l'accès à l'IRM était réservé aux spécialistes du sujet.

Mots clés. Imagerie par résonance magnétique. Lésion silencieuse. Découverte fortuite.

Anévrisme. Malformation artérioveineuse. Cavernome. Syndrome radiologique isolé.

\begin{abstract}
A coincidental finding is present in one out of 37 magnetic resonance imaging (MRI)-scans, besides white matter changes, brain microbleeds and silent infarcts. This prevalence increases after gadolinium enhancement, angiographic sequences, and with age.

The consequences of coincidental findings are sometimes potentially beneficial, for instance in case of a tumor or an isolated radiological syndrome. They are more often harmful because of psychological or social consequences, inappropriate management of cerebral arteriovenous malformations or when brain microbleeds or an aneurysm erroneously prevent neurologists from giving thrombolytic therapy. In some cases, there is uncertainty about the most
\end{abstract}


appropriate strategy, and the decision is based on a multidisciplinary approach considering the natural history of the disease, the risk of treatments, and patient's preference.

Physicians should bear in mind that coincidental findings are frequent and rarely lead to a specific management with a potential benefit for the patient. More frequently this finding generates anxiety for patients and relatives, creates difficulties with insurance companies and leads to an increased consumption of health resources. This should be anticipated, which would be easier if access to brain MRI were limited to brain specialists.

Key words. Magnetic resonance imaging. Silent lesion. Coincidental finding. Aneurysm. Arteriovenous malformation. Cavernoma. Isolated radiological syndrome. 


\section{Introduction}

La découverte fortuite d'anomalies lors d'une exploration en imagerie par résonance magnétique (IRM) est de plus en plus fréquente en raison de l'amélioration de la résolution des images [1]. Elle peut survenir à l'occasion d'une IRM demandée pour un motif clinique sans lien avec la découverte, ou plus rarement, chez des volontaires sains acceptant de participer comme témoins à des protocoles de recherche [1]. Dans une revue systématique des études publiées avant mai 2008, leur fréquence était de 2,7\%, soit 1 cas toutes les 37 IRM, en excluant les anomalies de la substance blanche, les infarctus silencieux et les microsaignements [2].

Les conséquences de ces découvertes sont parfois bénéfiques pour le patient lorsqu'elles permettent le traitement d'une lésion à risque évolutif grave. Elles sont aussi parfois délétères en raison des conséquences psychologiques et sociales qu'elles peuvent induire, ou d'attitudes thérapeutiques inadaptées alors que leur histoire naturelle est parfois méconnue, et que leur traitement expose à un certain risque.

L'objectif de cette revue est de déterminer la fréquence de ces découvertes fortuites, leurs conséquences, et la prise en charge spécifique la plus adaptée. Nous nous limiterons aux anomalies découvertes sur IRM.

\section{Circonstances révélatrices des anomalies intracrâniennes de découverte}

\section{fortuite}

Trois grandes circonstances amènent à ces découvertes :

- Une IRM faite dans un but de recherche chez des volontaires sains. Dans ce cas il y a obligatoirement l'accord d'un comité d'éthique. Le formulaire d'information doit expliquer au patient la possibilité de telles découvertes fortuites, et la conduite à tenir doit être prévue.

- Une IRM dont l'indication est validée et conforme aux recommandations professionnelles (accident vasculaire cérébral, maladie neurodégénérative, sclérose en plaques, épilepsie etc.). Ces découvertes ne sont pas évitables.

- Une IRM dont l'indication est discutable voire mauvaise (migraine mal prise en charge, vertiges de cause ORL, troubles fonctionnels etc). Ce sont les cas qui posent le plus de problème en pratique, car le patient n'a généralement pas été préparé à cette éventualité. L'examen n'a le plus souvent pas été demandé par un médecin spécialiste du sujet, et des informations erronées et alarmantes sont parfois données à tort. 


\section{Types d'anomalies intracrâniennes de découverte fortuite}

La prévalence globale des anomalies intracrâniennes de découverte fortuite chez des volontaires sains de 46 à 97 ans ayant des séquences IRM en haute résolution sans injection de gadolinium et sans temps angiographique est de l'ordre de 10\% (tableau I) [2]. Cette prévalence augmente en cas d'injection de gadolinium ou de séquences angiographiques [1]. Elle augmente aussi avec l'âge des personnes étudiées, une personne sur 3 âgée de plus de 65 ans ayant des anomalies sur l'IRM [2]. Dans une étude où les volontaires sains âgés de 71,5 à 78,9 ans étaient sélectionnés sur la base de nombreux critères d'exclusion, ce qui en fait des volontaires «super sains », seuls $111(22,1 \%)$ avaient une IRM normale [1]. Les 392 autres avaient 494 anomalies découvertes fortuitement dont 472 étaient considérées sans importance clinique, et seuls 22 ont justifié d'être explorées voire traitées [1].

\section{Les lésions cérébrales vasculaires}

Les infarctus silencieux (figure 1a) sont généralement de petits infarctus lacunaires profonds, chez des sujets âgés ou hypertendus. Ils sont présents sur la $1^{\text {er }}$ imagerie chez $20 \%$ des patients qui présentent un premier infarctus cérébral [3]. Ils sont le plus souvent le témoin d'une maladie des petites artères, associées à une hypertension artérielle, et à un risque plus élevé de déclin cognitif [4] mais pas de récidive vasculaire [5].

Les anomalies de la substance blanche (figure 1b) sont également fréquentes chez les sujets âgés asymptomatiques, d'autant plus qu'ils ont des facteurs de risque vasculaire. Elles sont aussi le témoin d'une atteinte chronique des petits vaisseaux intra-cérébraux [6].

Les micro-saignements (figure 1c) sont de petites lésions de moins de $10 \mathrm{~mm}$ de diamètre visibles uniquement en IRM sur des séquences écho de gradient $\mathrm{T} 2 *$ où elles apparaissent en hyposignal [7]. Leur prévalence est de 5\% chez des adultes sains, 34\% chez les patients ayant déjà eu un infarctus cérébral [7], et 60\% dans les hémorragies cérébrales, mais leur prévalence augmente avec la puissance magnétique et les nouvelles séquences d'écho de gradient [8]. Ils sont d'autant plus fréquents que le sujet est plus âgé, hypertendu, et présente des accidents vasculaires cérébraux (AVC) récidivants [7]. Des micro-saignements corticaux sont fréquents dans l'angiopathie amyloïde mais ils ne sont pas spécifiques [7]. Lorsqu'ils sont situés dans les noyaux gris centraux, le centre ovale, et la capsule interne, ils sont plutôt le témoin d'une affection des petits vaisseaux, chez des sujets hypertendus ou âgés [6]. 
Les anévrismes artériels cérébraux (figure 2) sont rapportés dans la méta-analyse de Morris et al [2] avec une prévalence de 0,35\% (intervalle de confiance à $95 \%$ de 0,13 à $0,67 \%$ ). Cette prévalence est vraisemblablement sous-évaluée car toutes les études inclues dans cette métaanalyse n'ont pas comporté de séquences vasculaires spécifiques en TOF (time-of-flight) [2]. Il est probable qu'en l'absence se séquence TOF seuls les anévrismes les plus volumineux ont été identifiés. Dans une étude japonaise ayant inclus 8857 patients consécutifs ayant eu une imagerie vasculaire en phase aiguë d'une ischémie cérébrale, 412 (4,7\%) avaient un anévrisme cérébral asymptomatique, dont le diamètre moyen était de 4,1 mm [9].

\section{Les lésions cérébrales non vasculaires}

La prévalence des lésions non tumorales est de $2 \%$ (IC 95\% 1,1\%-3,1\%), soit 1 cas toutes les 50 IRM, et celle des tumeurs est de 0,7\% (IC95\% 0,50\% - 0,98\%) soit 1 toutes les 143 IRM [2].

\section{Prise en charge des anomalies intracrâniennes de découverte fortuite.}

Situations dans lesquelles la découverte fortuite d'une anomalie intracrânienne peut être bénéfique.

La découverte fortuite d'une anomalie intracrânienne peut être utile lorsqu'elle permet de mettre le patient à l'abri d'une complication ultérieure, d'une évolutivité, ou de difficultés thérapeutiques lorsque la maladie sera plus évoluée. C'est probablement le cas dans les cas suivants :

- $\quad$ Les tumeurs à potentiel évolutif dans le cadre de l'espérance de vie supposée de la personne (méningiomes ou neurinomes chez des sujets jeunes, gliomes de bas grade). Ces circonstances sont toutefois assez rares.

- Le syndrome radiologique isolé («radiologically isolated syndrome, RIS »). Il s’agit de lésions de la substance blanche évocatrices de sclérose en plaques (figure 3) sur la base de leur taille, siège et morphologie, observées de façon fortuite sur une IRM faite pour d'autres raisons chez une personne n'ayant aucun signe de sclérose en plaques [10]. Environ 30 à $45 \%$ des personnes développeront des symptômes évocateurs de sclérose en plaques dans un délai de quelques années [10]. Les patients les plus à risque de conversion clinique sont ceux présentant des lésions médullaires, des bandes oligoclonales à la ponction lombaire, une activité inflammatoire marquée (lésions prenant le contraste et/ou une majoration de la charge lésionnelle au cours du temps) [10]. Ces patients doivent être suivis afin de pouvoir être traités 
sans délai si une évolution clinique se manifeste. Les traitements disease modifyers ayant un effet favorable dans les formes rémittentes de la maladie, se pose la question de savoir si un traitement introduit à ce stade permettrait de différer l'apparition des symptômes. En l'état actuel des connaissances, il n'y a pas de preuve d'un bénéfice à l'introduction d'un traitement de fond à ce stade purement radiologique et des études sont actuellement en cours [10-11].

\section{Situations dans lesquelles la découverte fortuite d'une anomalie intracrânienne peut amener à une prise en charge délétère}

Il est en revanche, des cas où la prise en charge de la pathologie, sur la base de ce que l'on connait des formes symptomatiques de la pathologie, s'avère délétère.

Le cas le plus spectaculaire concerne les malformations artérioveineuses (figure 4). La plupart de celles-ci sont maintenant de découverte fortuite. Il est tentant de proposer de la traiter car nous disposons d'un arsenal thérapeutique avec la chirurgie, la radiologie interventionnelle et la radiochirurgie. Toutefois, transposer aux formes n'ayant pas saigné nos connaissances des formes hémorragiques de ces malformations est hasardeux car leur histoire naturelle n'est pas connue. Toutes les malformations artérioveineuses ne saignent pas, et le traitement favorise parfois la rupture pendant les premiers mois. Une étude randomisée internationale a montré que les patients randomisés dans le groupe abstention avaient un risque 4 fois plus faible de décéder ou de présenter une hémorragie cérébrale dans les 33 mois de suivi (hazard ratio 0,27 ; intervalle de confiance à $95 \%$ de 0,14 à 0,54 ) par rapport à ceux qui étaient randomisés dans le groupe traité (chirurgie, radiochirurgie, radiologie interventionnelle, ou combinaison des techniques) [12]. Chez ces patients dont l'âge moyen était de 45 ans un suivi de 3 ans peut paraitre trop court pour répondre définitivement à la question. Néanmoins, le faible risque d'évènement dans le groupe non traité laisse supposer qu'il ne devrait pas y avoir de supériorité du traitement dans les 20 années suivant la randomisation [12].

Un autre exemple de prise en charge inappropriée serait de récuser une thrombolyse intraveineuse dans une ischémie cérébrale aiguë en présence de micro-saignements silencieux ou d'un anévrisme asymptomatique. Une étude française bi-centrique a montré que la tolérance de la thrombolyse était excellente chez les patients porteurs de micro-saignements et leur présence ne justifiait pas d'en récuser l'indication [13]. Toutefois, les patients ayant plus de 5 micro-saignements semblent à risque accru en cas de traitement thrombolytique ou anticoagulant [14-15]. Le risque est plus élevé avec un traitement anticoagulant oral au long cours qu'avec un traitement thrombolytique ponctuel. La présence de micro-saignements n'est pas une contre-indication à ces traitements, mais le rapport bénéfice risque devra être 
évalué, en particulier lors d'un traitement anticoagulant oral. Bien qu'il n'y ait jamais eu d'essai évaluant antivitamines $\mathrm{K}$ et anticoagulants oraux directs chez des patients porteurs de micro-saignements, il est préférable d'avoir recours aux anticoagulants oraux directs à chaque fois que possible en raison d'un risque d'hémorragie cérébrale plus faible [16].

\section{Situations incertaines}

Dans de nombreuses situations il y a une incertitude. Le bénéfice de la prise en charge d'un anévrisme qui n'a pas saigné, d'un cavernome, ou d'une lésion inflammatoire silencieuse, n'est pas prouvé. La décision doit reposer sur une évaluation précise du risque naturel et du risque des traitements, et la préférence du patient. Cela nécessite souvent une concertation multidisciplinaire.

\section{Les anévrismes artériels cérébraux}

Trois questions se posent en pratique lorsque l'on découvre un anévrisme artériel cérébral de façon fortuite :

Faut-il modifier la prise en charge de l'ischémie cérébrale lorsque l'IRM qui a découvert cet anévrisme a été effectuée pour une ischémie cérébrale ? Dans l'étude japonaise ayant inclus 8857 patients consécutifs ayant eu une imagerie vasculaire en phase aiguë d'une ischémie cérébrale [9], dont 412 (4,7\%) avaient un anévrisme cérébral asymptomatique de diamètre moyen de $4,1 \mathrm{~mm}$, le pronostic à 3 mois n'était pas moins bon chez les porteurs d'anévrismes, y compris en cas de traitement thrombolytique, anticoagulant ou antiplaquettaire. Cette donnée suggère qu'en phase aiguë la prise en charge de l'ischémie cérébrale ne doit pas être influencée par la présence d'un anévrisme asymptomatique $[9,17]$.

Faut-il traiter l'anévrisme? La décision repose sur la comparaison entre le risque de rupture spontanée en l'absence de traitement, le risque de complications liées au traitement, et l'influence sur la qualité de vie de se savoir porteur d'un anévrisme non traité [18]. Le risque de rupture dépend principalement de sa taille et de son siège. Les petits anévrismes saignent moins mais comme ils sont plus fréquents, dans la population la plupart des hémorragies sous arachnoïdiennes sont paradoxalement dues à la rupture de petits anévrismes [18]. Parmi les autres facteurs structurels exposant à un risque de rupture plus élevé figurent une paroi irrégulière, avec une «bulle» voire un deuxième sac au sein de l'anévrisme, et le ratio hauteur/profondeur [18]. Le tabagisme actif et l'hypertension artérielle sont également des facteurs de rupture. La décision de traiter ou pas doit mettre dans la balance le risque évalué de rupture, celui du traitement, l'espérance de vie du patient, et sa préférence. 
- Quel traitement proposer ? Le choix entre clipping et coiling dépend de l'angioarchitecture de l'anévrisme et des risques respectifs de chaque traitement. Lorsque le principe d'un traitement est retenu, le choix du traitement doit se faire au terme d'une concertation pluridisciplinaire envisageant les difficultés respectives de chaque traitement.

\section{Les hypersignaux non spécifiques de la substance blanche}

Ils sont favorisés par l'âge et les facteurs de risque vasculaire. Ils sont associés à un risque accru de démence [19]. Leur présence justifie de rechercher des facteurs de risque vasculaire et de les traiter en suivant les recommandations pour la prévention primaire.

\section{Conséquences de la découverte fortuite d'anomalies intracrâniennes}

Un médecin qui demande la réalisation d'une IRM doit avoir à l'esprit le risque de découverte d'anomalies fortuites, et que cette découverte ne va généralement rien changer au cours évolutif de la maladie qui a justifié l'examen. Traiter un anévrisme asymptomatique n'améliora pas les céphalées d'un migraineux. Découvrir une anomalie intracrânienne fortuite ne justifie que rarement sur une prise en charge spécifique bénéfique au patient. Au contraire, elle peut générer de l'anxiété non seulement chez les patients et leurs proches, mais aussi chez les médecins qui peuvent être ainsi incités à proposer des thérapeutiques invasives, en perdant de vue que l'histoire naturelle de ces anomalies ne justifie souvent pas le risque des traitements. Par ailleurs, ces personnes sont exposées au risque de refus d'assurances, et de consommation excessives et pas toujours justifiées de ressources médicales, en particulier les contrôles d'imagerie [20].

\section{Conclusion}

A chaque fois que l'on pratique 37 IRM cérébrales on découvre une lésion silencieuse. Il est important, en particulier quand l'indication de l'IRM n'est pas impérative, que le patient soit prévenu de cette éventualité. La découverte fortuite d'une anomalie intracrânienne expose plus souvent à des conséquences délétères qu'à un service rendu. C'est un risque auquel on est obligé d'exposer le patient lorsque l'indication de l'IRM est formelle et validée par un spécialiste. C'est en revanche un risque que l'on doit garder à l'esprit et dont le patient doit être informé dans les indications limites, voire discutables de cet examen, ou en recherche chez des volontaires sains. Une telle éventualité doit être anticipée, ce qui serait plus aisé si l'accès à l'IRM était réservé aux spécialistes du sujet. 
Conflits d'intérêt

Les auteurs déclarent ne pas avoir de conflit d'intérêt en lien avec cet article. 


\section{Points essentiels}

- La découverte fortuite d'anomalies lors d'une exploration IRM est observée 1 foiş tous les 37 examens - hors anomalies de la substance blanche, infarctus silencieux et micro-saignements-.

- Cette éventualité augmente en cas d'injection de gadolinium ou de séquences angiographiques, et avec l'âge des personnes.

- Les conséquences de ces découvertes sont rarement bénéfiques pour le patient

- Elles sont plus souvent délétères en raison des conséquences psychologiques et sociales qu'elles peuvent induire, ou d'attitudes thérapeutiques inadaptées.

- Une telle éventualité doit être anticipée et le patient doit en être informé. 


\section{Références}

[1] Boutet C, Vassal F, Celle S, Schneider FC, Barthélémy JC, Laurent B, et al. Incidental findings on brain magnetic resonance imaging in the elderly:the PROOF study. Brain Imaging Behav. 2017;11:293-9.

[2] Morris Z, Whiteley WN, Longstreth Jr. WT, Weber F, Lee YC, Tsushima Y, et al. Incidental findings on brain magnetic resonance imaging: systematic review and metaanalysis. Br Med J. 2009;339:b3016.

[3] Mounier-Vehier F, Leys D, Rondepierre P, Godefroy O, Pruvo JP. Silent infarcts in patients with ischemic stroke are related to age and size of the left atrium. Stroke. 1993;24:1347-51.

[4] Leys D, Hénon H, Mackowiak-Cordoliani MA, Pasquier F. Poststroke dementia. Lancet Neurol. 2005;4:752-9.

[5] Weber R, Weimar C, Wanke I, Möller-Hartmann C, Gizewski ER, Blatchford J, et al. Risk of recurrent stroke in patients with silent brain infarction in the Prevention Regimen for Effectively Avoiding Second Strokes (PRoFESS) imaging substudy. Stroke. 2012;43:350-5.

[6] Dichgans M, Leys D. Vascular Cognitive Impairment. Circ Res. 2017;120:573-91.

[7] Cordonnier C, Al-Shahi Salman R, Wardlaw J. Spontaneous brain microbleeds: systematic review, subgroup analyses and standards for study design and reporting. Brain. 2007;130:1988-2003.

[8] van Veluw SJ, Charidimou A, van der Kouwe AJ, Lauer A, Reijmer YD, Costantino I, et al. Microbleed and microinfarct detection in amyloid angiopathy: a high-resolution MRIhistopathology study. Brain. 2016;139:3151-62.

[9] Shono Y, Sugimori H, Matsuo R, Fukushima Y, Wakisaka Y, Kuroda J, et al. Safety of antithrombotic therapy for patients with acute ischemic stroke harboring unruptured intracranial aneurysm. Int J Stroke. 2018;13:734-42.

[10] Lebrun C. The radiologically isolated syndrome. Rev Neurol (Paris). 2015;171:698-706.

[11] De Stefano N, Giorgio A, Tintoré M, Pia Amato M, Kappos L, Palace J, et al. Radiologically isolated syndrome or subclinical multiple sclerosis: MAGNIMS consensus recommendations. Mult Scler. 2018;24:214-21.

[12] Mohr JP, Parides MK, Stapf C, Moquete E, Moy CS, Overbey JR, et al. Medical management with or without interventional therapy for unruptured brain arteriovenous malformations (ARUBA): a multicentre, non-blinded, randomised trial. Lancet. 2014;383:614-21. 
[13] Turc G, Sallem A, Moulin S, Tisserand M, Machet A, Edjlali M et al. Microbleed Status and 3-Month Outcome After Intravenous Thrombolysis in 717 Patients With Acute Ischemic Stroke. Stroke. 2015;46:2458-63.

[14] Wilson D, Ambler G, Shakeshaft C, Brown MM, Charidimou A, Al-Shahi Salman R, et al. Cerebral microbleeds and intracranial haemorrhage risk in patients anticoagulated for atrial fibrillation after acute ischaemic stroke or transient ischaemic attack (CROMIS-2): a multicentre observational cohort study. Lancet Neurol. 2018;17:539-47.

[15] Charidimou A, Boulouis G, Shams S, Calvet D, Shoamanesh A; International METAMICROBLEEDS Initiative. Intracerebral haemorrhage risk in microbleed-positive ischaemic stroke patients with atrial fibrillation: Preliminary meta-analysis of cohorts and anticoagulation decision schema. J Neurol Sci. 2017;378:102-9.

[16] López-López JA, Sterne JAC, Thom HHZ, Higgins JPT, Hingorani AD, Okoli GN, et al. Oral anticoagulants for prevention of stroke in atrial fibrillation: systematic review, network meta-analysis, and cost effectiveness analysis. BMJ. 2017;359:j5058.

[17] Goyal N, Tsivgoulis G, Zand R, Sharma VK, Barlinn K, Male S, et al. Systemic thrombolysis in acute ischemic stroke patients with unruptured intracranial aneurysms. Neurology. 2015;85:1452-8.

[18] Rinkel GJE. Management of patients with unruptured intracranial aneurysms. Curr Opin Neurol. 2019 (in press).

[19] Debette S, Markus HS. The clinical importance of white matter hyperintensities on brain magnetic resonance imaging: systematic review and meta-analysis. BMJ. 2010;341:c3666.

[20] Illes J, Borgelt E. Brain imaging: incidental findings: in practice and in person. Nat Rev Neurol. 2009;5:643-4. 
Tableau I. Prévalence des anomalies intracrâniennes de découverte fortuite (à l'exclusion des hypersignaux de la substance blanche, des micro-saignements et des infarctus silencieux) modifié d'après la méta-analyse de Morris et al. ${ }^{1}$

\begin{tabular}{|c|c|c|}
\hline Type de lésion & $\begin{array}{c}\text { IRM nécessaires pour } \\
\text { trouver } 1 \text { cas }\end{array}$ & $\begin{array}{c}\text { Prévalence / } \\
1000 \text { IRM }\end{array}$ \\
\hline Tumeurs (tous types) & 143 & $7,0(4,7-0,9,8)$ \\
\hline Méningiomes & 345 & $2,9(1,3-5,1)$ \\
\hline Gliomes de bas grade & 2000 & $0,5(0,2-0,9)$ \\
\hline Neurinome acoustique & 3333 & $0,3(0,1-0,6)$ \\
\hline Lipome & 2500 & $0,4(0,2-0,7)$ \\
\hline Kyste épidermoïde & 3333 & $0,3(0,1-0,6)$ \\
\hline Malformations vasculaires & 201 & $5,0(4,1-6,0)$ \\
\hline Anévrisme & 286 & $3,5(1,3-6,7)$ \\
\hline Cavernome & 325 & $1,6(1,0-2,3)$ \\
\hline Malformation artério-veineuse & 2000 & $0,5(0,1-1,0)$ \\
\hline Lésions inflammatoires & 1504 & $0,7(0,4-1,1)$ \\
\hline Certaines & 1667 & $0,6(0,2-1,5)$ \\
\hline Possibles & 3333 & $0,3(0,0-0,7)$ \\
\hline Kystes & 194 & $4,6(3,7-5,7)$ \\
\hline Arachnoïdien & 200 & $5,0(2,1-8,7)$ \\
\hline Colloïdes & 2500 & $0,4(0,1-0,7)$ \\
\hline Autres anomalies & 173 & $7,0(4,7-9,8)$ \\
\hline Malformation de Chiari type I & 417 & $2,4(0,4-5,8)$ \\
\hline Hydrocéphalie & 1000 & $1,0(0,3-1,9)$ \\
\hline Collection extra-axiale & 2500 & $0,4(0,1-0,7)$ \\
\hline Toutes anomalies confondues & 45 & $22,3(20,3-24,5)$ \\
\hline
\end{tabular}




\section{Figures.}

Figure 1. Lésions parenchymateuses silencieuses, d'origine vasculaire, en rapport avec une micro-angiopathie cérébrale. Infarctus silencieux (1a). Anomalies de la substance blanche (1b). Micro-saignements (1c).

Figure 2. Anévrisme de l'artère cérébrale moyenne de découverte fortuite.

Figure 3. Syndrome radiologique isolé ayant évolué vers une sclérose en plaques dans les 2 ans.

Figure 4. Malformation artério-veineuse de découverte fortuite. 


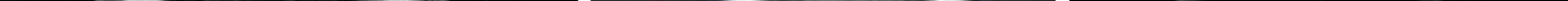




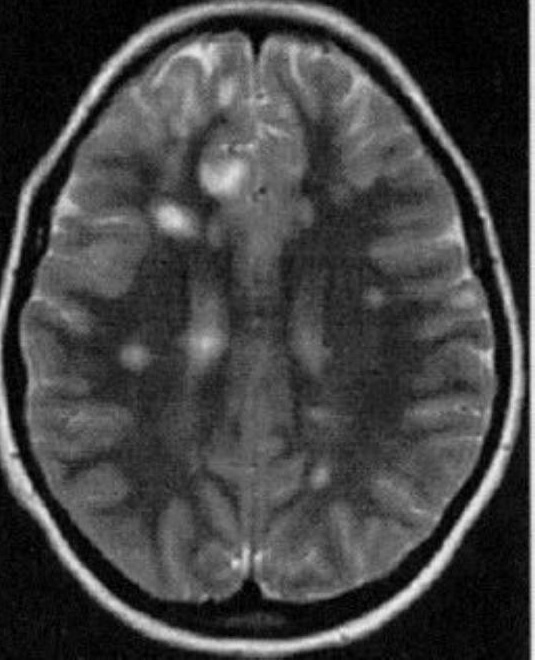




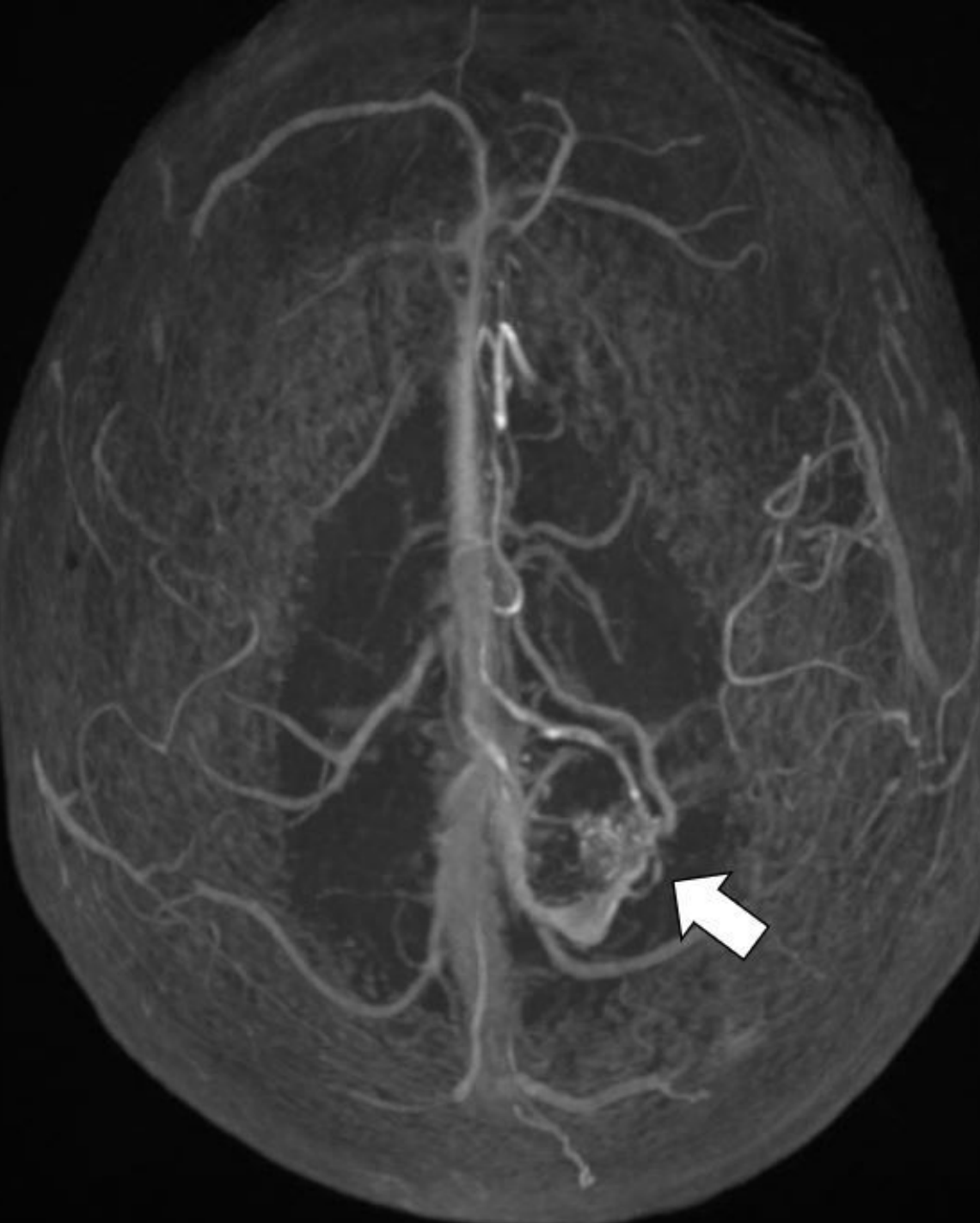

